\title{
A Median Filtering Method for Pulse Noise Detection
}

\author{
Yamei Zhang \\ Xi'an Peihua Universty \\ Xi'an, China, 710125 \\ e-mail: 312413420@qq.com
}

\begin{abstract}
This paper presents a method for detecting image impulse noise and using median filter to filter out impulse noise according to the test results. The method will contain the subspace of the subimage of the impulsive noise, and the kernel function is mapped into a hypersphere in the high dimensional space, and the radius $R$ of the sphere and the corresponding spherical vector a are calculated. The median filter method is used to filter the detected noise points. The proposed algorithm is more accurate in judging noise, and the way to filter noise is more reasonable. The applicable image range is more extensive and has better filtering performance.
\end{abstract}

Keywords-Impulse Noise; Noise Detection; Kernel Function; Median Filter (MF)

\section{INTRODUCTION}

In this paper, the use and analysis of nuclear methods are presented in this paper. Digital image is an important source of information for many fields of science students, such as biology and astronomy, and so on, but the actual collected images often because image acquisition system, transmission medium and imaging system is not perfect and the introduction of different levels of noise, so the effective noise removal work has become a critical part of the image processing, because a lot of work, such as edge detection, image segmentation, character recognition, etc.) are largely dependent on the stand or fall of noise removing. When the image is polluted by noisy circuit or electronic induced noise in the encoding and transmission, the noise of the image drop is mainly pretz-salt noise (i.e. positive and negative impulse noise).

The main method of filtering noise is to make image filtering. The requirement of image filtering is to remove the noise in the image and to keep the details of the image. In common filtering algorithms, mean filtering, median filtering (MF) and adaptive filtering (AMF) are widely used. When the probability of noise pollution is lower than the mean filter, median filter is better than mean filtering. When the probability of noise pollution is greater, the mean filtering is better than the median filter. The median filter (MF) is more effective for images with less noise density than 0.2. When the noise density is higher than 0.2, the adaptive filter (AMF) can be used to filter the image noise. But the commonly used filtering algorithm, while filtering the noise image, will blur the detail edge of the image and lose some important information of the image. In this paper, a new method of median filtering based on image pulse noise detection is proposed, which can effectively detect and remove noise points, and keep the original information of the image intact. Compared with other algorithms, the algorithm proposed by the paper is more accurate in the judgment of noise, and the method of filter noise is more reasonable, and the applicable image range is wider and has better filtering performance.

\section{STANDARD MEDIAN FILTERING}

The median filter is a typical sort based nonlinear filter, which was proposed by Turky in 1971. The standard median filter can be completed in the following steps:

1) The template is roamed in the image, and the center of the template overlaps with one pixel in the figure

2) To read the gray value of each corresponding pixel in the template

3) Align these gray values from small to large

4) Find out the middle of these values

5) Assign this intermediate value to the pixel of the corresponding template center position.

Set $\left\{x_{i j}, \quad(i, j) \in I^{2}\right\}$ to represent the grayscale value of each point in the digital image, and the filter window is $\mathrm{A}$ two-dimensional median filter of A, which can be defined as :

$$
\begin{gathered}
\mathrm{y}_{\mathrm{ij}}=\operatorname{Med}\left\{\mathrm{x}_{\mathrm{ij}}\right\}=\operatorname{Med}\left\{\mathrm{x}_{(\mathrm{i}+\mathrm{r})}(\mathrm{j}+\mathrm{s})\right. \\
\left.(\mathrm{r}, \mathrm{s}) \in \mathrm{A},(\mathrm{i}, \mathrm{j}) \in \mathrm{I}^{2}\right\}
\end{gathered}
$$

The size of the neighborhood determines the median value in the number of values, and the shape of the window determines the value of the element calculation in the geometry space. For two-dimensional images, the shape of window A can be rectangular, circular, cross, etc. Its center is generally located at the processing point.

Standard median filter to the performance of the impulse noise are greatly influenced by the filter window size, to suppress the image noise and protect the details there is contradiction from two aspects: a small window filtering, can better protect the image details, but the ability to filter out noise could be limited; Filter window is big, can strengthen the noise suppression ability, but the protection of detail ability will abate, sometimes filter to the image of some fine lines, sharp edges and other important details, which damage image geometric structure. This kind of contradiction is obvious when the noise interference in the image is bigger.

\section{A NEW IMAGE NOISE DETECTION ALGORITHM}

In order to filter the impulse noise at the same time to preserve the detail of the image, this paper proposes a new image impulse noise detection algorithm: based on the 
principle of a classification method is image impulse noise detection technology. This method is used to reduce noise image and effectively preserve the image details.

\section{A. The Idea of an Algorithm}

Class classification method is also known as data description, a special kind of classification method, is used to describe the characteristics of the existing data, and to judge the new data, whether the identified categories belong to the original data. There are many solutions to single class classification problems. Schlkopf et al. extended SVM to a class of problems called SVM one-class. D.M.J. Tax establishes the support vector data description (SVDD) to map the sample space to the nuclear space using the gaussian kernel function to find a sphere that can contain all the training data in the nuclear space. If the test sample is in this high-dimensional sphere, then it is considered normal. Otherwise it would be considered an anomaly. These models have been widely used in the fields of text classification, handwriting recognition and image processing.

A class classification method for image noise detection can effectively solve the contradiction between noise reduction and preserving image details. One of the direct methods of classification problems is to include a sample of the entire sample with a supersphere with a minimum volume. For the sample set:

$$
X=\{X 1, X 2, X 3, \ldots \ldots, X n\}
$$

The radius of the minimum sphere corresponding to the total sample of the sample is set to $\mathrm{R}$, and the corresponding spherical vector is a, then $R$ and a satisfies the following optimization equation:

$$
\min \mathrm{Q}(\mathrm{R})=\mathrm{R} 2
$$

$$
\text { s.t. } \mathrm{R} 2-(\mathrm{Xi}-\mathrm{a}) \mathrm{T}(\mathrm{Xi}-\mathrm{a}) \geqslant 0, \mathrm{i}=1,2,3, \cdots, \mathrm{n}
$$

The Lagrange function is established by formula (3) and (4) as in:

$$
\begin{aligned}
& \mathrm{L}(\mathrm{R}, \mathrm{a}, \alpha)=\mathrm{R} 2 \cdot \sum_{i=1}^{n} a_{i}-\{\mathrm{R} 2-(\mathrm{Xi}-\mathrm{a}) \mathrm{T}(\mathrm{Xi}-\mathrm{a})\} \\
& \alpha \mathrm{i} \geqslant 0, \mathrm{i}=1,2,3, \cdots, \mathrm{n}
\end{aligned}
$$

So if I take $\mathrm{L}(\mathrm{R}, \mathrm{a}$, alpha) to $\mathrm{R}$, I take the minimum of a, so I take the equation (5) with respect to R, I take the partial derivative with respect to a, and I make them 0 , For a quick as in (7):

$$
\sum_{i=1}^{n} a_{i}=1
$$

Make a little change, for a quick optimization equation:

$$
\operatorname{maxL}=\sum_{i=1}^{n} a_{i}\left(X_{i} \cdot X_{j}\right)-\sum_{i=1}^{n} \sum_{j=1}^{n} a_{i} a_{j}\left(X_{i} \cdot X_{j}\right)=1
$$

$$
\begin{aligned}
& \text { s.t. } \sum_{i=1}^{n} a_{i}=1 \\
& \alpha \mathrm{i} \geqslant 0, \mathrm{i}=1,2,3, \cdots, \mathrm{n}
\end{aligned}
$$

Similar to Support Vector machine (SVM), according to the KKT conditions, only part of the alpha alpha I > 0 , it is with this part of the alpha I the sample points determines the corresponding closed super sphere radius and centre, for and Support Vector (Support Vector, SV) phase difference, we call these sample points Support Object (the Support Object, $\mathrm{SO})$. After the kernel function is introduced, the corresponding discriminant function is:

$$
\begin{aligned}
& \alpha_{\text {i K }\left(\mathrm{K}, X_{i}\right)} \\
& f(\mathrm{Z})=\mathrm{K}(\mathrm{Z}, \mathrm{Z})-2 \sum_{i=1}^{n} a_{\mathrm{i} K}\left(\mathrm{~K}, X_{i}\right)+\sum_{i=1}^{n} a_{i} a_{j} \mathrm{~K}\left(X_{i} \cdot X_{j}\right)
\end{aligned}
$$

\section{B. Algorithm Design and Implementation - Detection and Filtering of Image Pulse Noise}

Detection of noise points in the impulsive noise image, can be made from a kind of classification method to do the following treatment: first generate a sliding window of $3 * 3$ matrix, the matrix can be the window on the entire image from top to bottom, from left to right sliding. In the process of each movement of the window matrix, we selected 9 points of the window matrix as training samples: $X=\{X 1$, $\mathrm{X} 2, \ldots \mathrm{X} 9\}$, and then use the gaussian kernel function to train these samples to generate a super sphere with a radius $\mathrm{R}$. Then use the formula of center of test sample window matrix $\mathrm{Z}$, testing it to a distance of its $\mathrm{f}(\mathrm{Z})$, according to the $\mathrm{f}(\mathrm{Z})$ and $\mathrm{f}(\mathrm{XS})$, the relationship between the detection window with discriminant formula is the center of the matrix for the noise points. If there is a positive threshold value greater than set in the test result, it is concluded that the test sample window matrix center point $\mathrm{Z}$ is noise. If the test result is less than the set threshold epsilon, there is no noise in this window matrix. When the noise is detected, the median filter is used to filter the image of the window matrix, and the filtered results are added to a new matrix with the same size as the original image. Iterate over this process until the complete image is processed.

\section{EXPERIMENT AND RESULTS}

On the computer of PC P4 T2310 1.86G, 2GRAM, Inte182865G graphics card, with Mat Lab 6.5 as the development environment, we realized the noise detection and noise reduction in the noise image we proposed. From the experimental results, the proposed method is effective and ideal. 
This paper deals with the medical organ map as shown in the figure below. Fig. 1(a) is the original image, and after adding $20 \%$ of the pulse noise to the original image, noise image as in Fig.1 (b) is obtained. For noise image as in Fig.1 (b), the noise reduction is processed through the above algorithm. A 3 by 3 sliding window matrix is generated here, from top to bottom in noise image as in Fig.1 (b), and from left to right, different subimages can be obtained. Each time you move, you get 9 pixels of information. Here we take three properties of each pixel. As a training sample $X=\{X 1$, $\mathrm{X} 2, \ldots \mathrm{X} 9$ \} can generate a 9 by 3 matrix, each representing a pixel's horizontal coordinate, ordinate, grayscale value. The training sample $\mathrm{X}$ and the test sample $\mathrm{Z}$ can be:

$$
X=\{x i, y i, g r a y(x i, y i)\}
$$

$$
\mathrm{i}=1,2 \ldots 9, \mathrm{Z}(\mathrm{Zx}, \mathrm{Zy} \text { gray }(\mathrm{Zx}, \mathrm{Zy}))
$$

Next to noise image as in Fig. 1(b), by using classification algorithm, the training samples for training, we take the first three lines of noise image as in Fig. 1 (b) as the training sample, all starting from the fourth row as test samples. This paper takes the medical organ image as an example, and the denoising effect of this method is compared with the standard median filter. The evaluation criteria are peak signal-to-noise ratio (PSNR), namely:

$$
\begin{aligned}
& \text { MSE }=\frac{\sum_{i=1}^{n} \sum_{j=1}^{m}\|F(i, j)-G(i, j)\|^{2}}{N M} \\
& \text { RMSE }=\sqrt{M S E} \\
& \text { PSNR }=20 \log \left\{\frac{255}{\mathrm{RMSE}}\right\}
\end{aligned}
$$

$\mathrm{M}$ and $\mathrm{N}$ are respectively rows and columns of images, MSE mean variance.

TABLE I. DENOISING EXPERIMENT DATA

\begin{tabular}{|c|c|c|c|}
\hline $\begin{array}{c}\text { The noise } \\
\text { density P }\end{array}$ & $\begin{array}{c}\text { The noise } \\
\text { of image }\end{array}$ & $\begin{array}{c}\text { Standard median } \\
\text { filtering }(\mathbf{9} * \mathbf{9})\end{array}$ & $\begin{array}{c}\text { In this } \\
\text { paper, the } \\
\text { filter }\end{array}$ \\
\hline 0.1 & 37.179 & 34.624 & 37.989 \\
\hline 0.2 & 34.196 & 34.137 & 37.457 \\
\hline 0.4 & 31.076 & 33.385 & 36.872 \\
\hline 0.6 & 29.312 & 32.669 & 36.395 \\
\hline 0.8 & 27.966 & 32.073 & 35.767 \\
\hline 1.0 & 27.104 & 30.635 & 33.643 \\
\hline
\end{tabular}

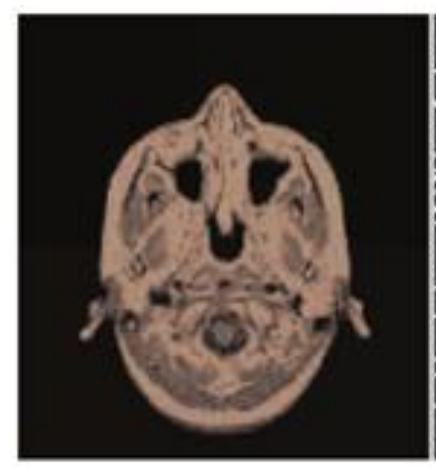

(a) original image

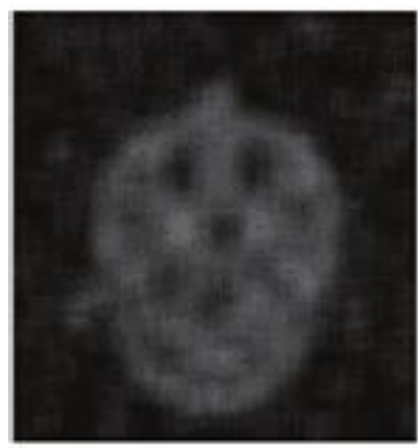

(c) mean filtering image

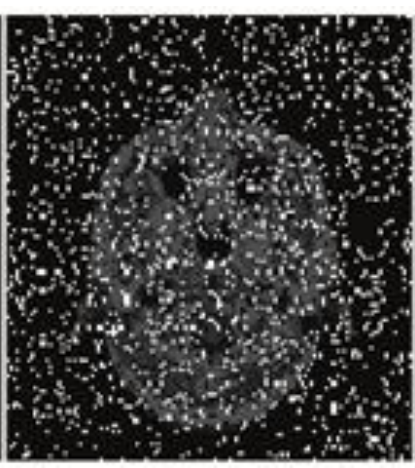

(b) noise image $(\mathrm{p}=0.2)$

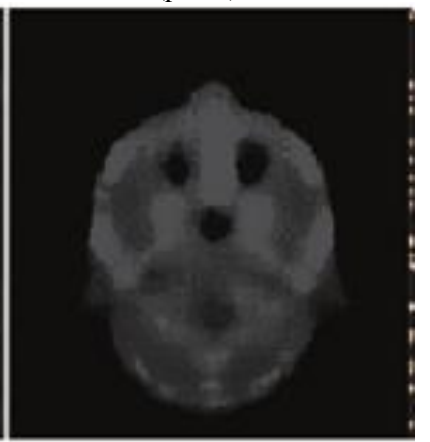

(d) median filter image

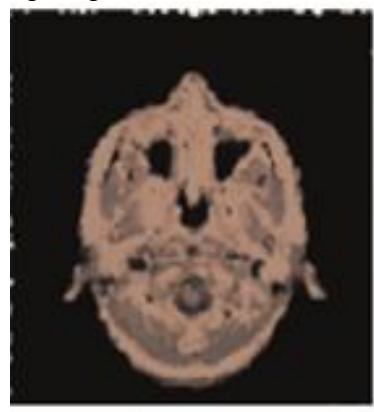

(e) filtering of this article

Figure 1. The original image and its experimental results.

From TABLE 1 and Figure 1 image corresponding to the filter, this paper puts forward the impulse noise detection algorithm is better than standard median filter effect, at the same time of filter impulse noise can be a very good reserve the detail of the image.

In this paper, based on the type of algorithm on the basis of classification problems using median filtering algorithm, through the image in the two-dimensional space nucleation for super sphere in high dimensional space, whether testing center of sliding window for noise, and filtering out noise through point by point by using median filtering algorithm. This method can not only effectively filter pulse noise but also keep the edge detail of image well. This paper illustrates the effectiveness of the method, which is of great significance to the research of digital image processing. 


\section{CONCLUSION}

By establishing kernel function, it has more accurate characteristics of noise recognition, which can accurately reflect the distribution characteristics of actual data. Compared with the standard median filter, the noise detection method can effectively filter out the pulse noise and keep the edge details of the image well. This paper illustrates the effectiveness of the method, which is of great significance to the research of digital image processing.

\section{ACKNOWLEDGMENT}

The project originates from Xi 'an PeiHua University" Study on intelligent identification and automatic diagnosis of ECG”(No. PHKT17053).

\section{REFERENCES}

[1] S. Theodoridis and K. Koutroumbas. Pattern Recognition. Academic Press, 2003.

[2] A. Smola. On a kernel-based method for pattern recongnition, regression, approximation and operator inversion.Algorithmica,22: 211-231,1998.

[3] N.Aronszajn.Theory of reproducing kernels. Transactions of the American Mathematical Society, 68:337-404,1950.

[4] 20.Aubert G, Kornprobst P.Mathematical Problems in Image Processing: Partial Differential Equations and the Calculus of
Variations [J]. Applied Mathematical. Sciences, Springer- Verlag, 2001;147.

[5] Muller K R, Mika S, Ratsch G, et al. An Introduction to Kernel-based Learning Algorithms. IEEE Trans. on Neural Networks [J]. 2001, 12 (2): $181-201$

[6] Tax D, Duin R. Combining One-class Classifiers. In: Kittler J, Roli F (Eds.). Multiple Classifier Systems, Proceedings Second International Workshop MCS 2001 [J] (Cambridge, UK, July), Lecture Notes in Computer Science, 2001, 2096:299-308

[7] [3]Tax D, Duin R. Data Domain Description Using Support Vectors [J]. Proc. European Symposium Artificial Neural Networks 1999:251-256

[8] Bengtsson M, Kock S.Cooperation and Competition in Relationships Between Competitors in Business Networks. Journal of Business \&Industrial Marketing [J], 1999, 14(3):178-193

[9] Bellman R E. Adaptive Control Processes: A Guided Tour [M]. Princeton University Press, Princeton, 196

[10] GONZALEZ R C, WOODS R E. Digital image processing [M]. Englewood Cliffs, NJ : Prentice Hall, 1992:241-243.

[11] ARAKAWA K. Median filter based on fuzzy rules and its application to image restoration [J]. Fuzzy Sets and Systems, 1996, 77( 1 ) : 3-13.

[12] OPRAK A, GULER I. Impulse noise reduction in medical images with the use of switch mode fuzzy adaptive median filter [J]. Digital Signal Processing,2007, 17(4) :711-713.

[13] TOPRAK A, GULER I. Suppression of impulse noise in medical images with the use of fuzzy adaptive median filter [J]. Journal Medical Systems, 2006, 30 ( 6 ) : 465- 471.

[14] Three-dimensional weighting reconstruction algorithm for circular cone-beam CT under large scan angles 